\title{
Éducation et économie de marché en Suède
}

Leif Brettell et Sverker Lindblad

Traducteur : Patricia Vrinat

\section{(2) OpenEdition}

\section{Journals}

Édition électronique

URL : http://journals.openedition.org/ries/3178

DOI : 10.4000/ries.3178

ISSN : 2261-4265

Éditeur

Centre international d'études pédagogiques

Édition imprimée

Date de publication : 1 septembre 1997

Pagination : 115-125

ISSN : 1254-4590

Référence électronique

Leif Brettell et Sverker Lindblad, «Éducation et économie de marché en Suède », Revue internationale d'éducation de Sèvres [En ligne], 15 | 1997, mis en ligne le 04 juillet 2013, consulté le 02 mai 2019. URL : http://journals.openedition.org/ries/3178; DOI : 10.4000/ries.3178

Ce document a été généré automatiquement le 2 mai 2019

(c) Tous droits réservés 


\title{
Éducation et économie de marché en Suède
}

\author{
Leif Brettell et Sverker Lindblad
}

Traduction : Patricia Vrinat

\section{Aperçu du système scolaire suédois}

1 Après 1950, l'éducation s'est développée dans les «États providence » de l'Europe moderne. L'expansion fut différente selon les pays et selon leur histoire, leur culture, leur industrie, leur politique et leur économie. Aujourd'hui, on a de nouvelles exigences vis-àvis de l'éducation. Maintenant, l'industrie et l'État considèrent l'éducation comme un outil pour survivre et progresser dans un monde international de plus en plus compétitif. On voit émerger des perspectives et des modèles de changement différents, comme le fait que les écoles et les enseignants doivent rendre des comptes ou le fait de donner davantage de choix aux parents et aux élèves. On voit aussi s'établir de nouvelles relations entre l'État et les autorités locales.

Comment le système éducatif relève-t-il le nouveau défi de devenir la «meilleure école d'Europe »? Pour comprendre la situation en Suède, nous allons décrire les problèmes principaux qui sont au cœur du système éducatif suédois d'hier et d'aujourd'hui.

3 En 1842, une loi a introduit l'éducation publique élémentaire en Suède. Cette éducation dépendait de l'État, mais dès le départ la gestion en revenait aux autorités locales et à l'Église. A la même époque, on a introduit un système parallèle, avec des lycées gérés directement par l'État. L'école publique s'est développée au début de ce siècle et s'est étendue à d'autres domaines, avec la création d'écoles à but professionnel ou celle d'écoles spécialisées.

4 Au cours de la Deuxième Guerre mondiale, à l'époque des horreurs et des ravages du fascisme, une commission éducative suédoise a esquissé un nouveau système scolaire démocratique. On a mis en place le nouveau système dans les années soixante; il comprenait une école unique avec neuf niveaux allant de 7 à 16 ans et une école 
secondaire supérieure allant de 16 à 18 ou 19 ans. Dans cette nouvelle école secondaire, les études théoriques et professionnelles étaient mêlées et les élèves pouvaient choisir selon leurs intérêts et capacités à partir du niveau 9 . Entre $85 \%$ et $95 \%$ des élèves arrivaient au bout des deux ou trois années du programme du cycle terminal. En même temps, l'éducation pour les adultes s'est répandue et elle est devenue une alternative et une source de compensation.

Dans l'école unique, le groupe classe était formé d'élèves hétérogènes à partir de la première classe et pouvait, en principe, monter sans changement jusqu'au niveau 9. Pour atteindre un bon niveau de résultats pour l'ensemble des élèves de ces classes hétérogènes, de nouveaux moyens ont été attribués, surtout pour venir en aide aux enfants lents et pour favoriser leur intégration. Des instituteurs intervenaient dans les niveaux 1 à 6 , alors que pour les niveaux 7 à 9 on faisait appel à des professeurs spécialisés dans deux ou trois matières.

6 Pendant cette même période, de nouvelles écoles modernes ont été construites, la formation des enseignants s'est développée, tout comme les nouveaux matériaux d'enseignement et les ressources pour la direction des écoles. Dans le nouveau programme national de 1980, le principe de la classe hétérogène est resté, mais on a insisté sur le fait que l'éducation doit être planifiée à partir des aptitudes réelles de chaque enfant. La même loi a interdit le regroupement par capacités de manière générale. A la fin des années quatre-vingt, on a mis en place une nouvelle réforme qui concernait la formation des enseignants; ils devaient avoir des connaissances plus larges et plus approfondies pour chaque matière. Maintenant, on forme deux catégories d'enseignants, une pour les niveaux 1 à 7 et l'autre pour les niveaux 7 à 9 , ce qui veut dire que ces deux catégories se superposent et qu'elles sont capables de coopérer davantage et d'être plus performantes.

7 La Suède a maintenant une population de 8,8 millions d'habitants. Au cours des années cinquante et des années soixante, des immigrants sont venus de Finlande et du sud de l'Europe pour travailler, puisque la Suède manquait de main-d'œuvre. Au cours des vingt dernières années, environ 40000 réfugiés sont arrivés en Suède chaque année. De nos jours, il y a une société multiculturelle en Suède, avec environ un million de citoyens qui sont nés à l'étranger ou qui ont des parents étrangers. Au cours des années quatre-vingt, dans le cadre de la classe hétérogène, on a accordé aux enfants d'immigrants des ressources pour l'apprentissage du suédois comme deuxième langue et langue d'adoption.

\section{Un nouveau contexte pour l'éducation suédoise}

8 De 1960 à 1980, l'État providence suédois s'est développé très rapidement. À partir des années cinquante, les autorités locales avaient petit à petit étendu leurs activités. Les impôts locaux avaient atteint un niveau maximum de $30 \%$ dans la plupart des villes au début des années quatre-vingt. Au cours de ces années, le secteur public a été remis en question, pour des raisons à la fois économiques et idéologiques. Beaucoup d'hommes politiques parlaient de cette façon :

«Le secteur public ne peut pas continuer à croître, l'industrie s'est ralentie et le poids de la taxation et de la réglementation est trop lourd pour les entreprises et les familles ».

9 Au cours de la même période, les compétences des autorités locales s'étaient élargies dans les domaines économiques et administratifs concernant la protection sociale, les besoins 
des personnes âgées et l'éducation. L'État se chargeait toujours de la distribution des financements d'une manière traditionnelle et bureaucratique et décidait des objectifs et de l'organisation.

Il fallait créer un système de subventions plus flexible et efficace. La solution adoptée fut la décentralisation. Le résultat fut que de nouvelles relations se forgèrent entre l'État et les autorités locales en ce qui concerne l'éducation. Depuis 1991, l'État a seulement le pouvoir de décider du programme national et de l'évaluation du système, il ne s'occupe donc plus que des objectifs et des résultats. Aujourd'hui, chaque autorité locale est pleinement responsable de la distribution des financements, de l'organisation, de l'embauche et du licenciement des enseignants et des chefs d'établissements, etc. Dans le même temps, un système de chèque-éducation a été introduit, à la fois pour les écoles publiques et pour les écoles privées, pour introduire un système de choix et de compétition entre les écoles. Ainsi, dans la plupart des deux cent quatre-vingt-trois autorités locales, les écoles ont dû travailler avec des subventions réduites. Dans le passé, c'était l'État qui décidait et réglementait le travail des enseignants. Aujourd'hui, ce sont les autorités locales qui assurent le salaire des enseignants définis à partir de conventions locales où la notion de mérite a été introduite.

11 Pendant cette période de réforme intense, le gouvernement a décidé d'adopter un nouveau programme national, introduit en 1994. En fait, le système suédois a changé très nettement. D'un système centralisé avec des règles strictes, il est devenu un système décentralisé, axé sur des objectifs. Chaque école doit maintenant planifier et mettre en place son propre programme à partir des objectifs nationaux. Mais, aujourd'hui, on demande très ouvertement des changements pour avoir un enseignement et un apprentissage plus efficaces. L'objectif national est toujours l'équité et les classes restent hétérogènes dans la plupart des cas. Mais, maintenant, il est clair qu'il risque d'y avoir moins d'équité, puisque le système utilise la loi du marché avec des chèques-éducation, la compétition et la liberté de choix. En 1997, on n'est pas convaincu que ce nouveau système d'éducation produise de meilleurs résultats pour les élèves. Ce que l'on entend le plus dans le débat aujourd'hui est que les résultats des élèves ne s'améliorent pas et sont très souvent en baisse. La réduction du budget et les incertitudes au plan local et au niveau de chaque école, en ce qui concerne les objectifs et l'organisation, créent des problèmes.

\section{Politique d'éducation et transformations de l'État providence}

Dans une certaine mesure, on peut considérer la «restructuration » sous sa forme de décentralisation et l'introduction de la loi du marché avec tout ce qui en découle comme un mouvement mondial, aussi bien en ce qui concerne l'économie que l'éducation. Cependant, la restructuration n'implique pas la même chose aux États-Unis, en France, en Grande-Bretagne et, bien sûr, en Suède. Il est donc important de prendre en considération ce qui est propre au cas suédois en ce qui concerne la direction des écoles. Qu'est devenu le contrôle démocratique de l'éducation par l'État providence d'autrefois ? Quelles sont les raisons des tentatives de déréglementation et de l'emploi de la notion de marché dans l'éducation en Suède ? Comment cela a-t-il fonctionné ? Va-t-on vers une période où l'on ignore le marché dans les écoles uniques en Suède? 
13 La Suède s'est transformée très rapidement et une société traditionnelle agraire, s'est vue remplacée par une société industrialisée avancée. Depuis les années vingt, un modèle spécifique de protection sociale s'est mis en place en Suède, basé sur la collaboration entre le capital et le travail et donnant lieu à un certain nombre de réformes sociales, ainsi qu'à l'expansion du secteur public.

14 C'est le développement concordant de la démocratisation et de l'industrialisation qui est le fondement du modèle suédois. Ainsi, deux élites parallèles ont émergé. La première, basée sur les associations du monde du travail, dominait le monde politique et la seconde, basée sur la propriété privée, dominait le monde économique. L'idée justifiant le compromis historique entre ces deux élites était que l'on pouvait concilier les intérêts conflictuels et surmonter les problèmes sociaux par le biais de l'expansion du secteur public. On a adopté un certain nombre de réformes basées sur des concepts tels le centralisme, l'universalisme et le consensus. Les problèmes sociaux devaient être réglés par des mesures standardisées, décrétées par un pouvoir central.

15 Au cours des années soixante-dix, différentes tendances politiques ont attaqué ce modèle. La décentralisation, l'individualisation et la polarisation sont devenues des tendances plus fortes dans la politique. D'une certaine façon, c'était le retour aux contradictions des années vingt, mais avec un capitalisme plus fort et mieux organisé. L'un des moyens de comprendre ces changements est de lire le rapport d'une commission d'État sur le pouvoir et la démocratie. Vers la fin des années quatre-vingt, le gouvernement a chargé cette commission d'étudier la répartition du pouvoir et l'influence des citoyens dans la société suédoise. La conclusion principale de son président, Olof Peterson ${ }^{1}$, était la suivante :

16 «On est à la fin d'une période caractérisée par une forte expansion du secteur public et par des conventions collectives centralisées, fondées sur un compromis historique entre le monde du travail et le capital, sur l'organisation sociale et sur des solutions standard décidées par un pouvoir central.

17 «Les institutions de l'État providence, qui étaient fondées sur des principes prédominants à l'époque où l'industrialisation et la démocratisation se sont développées, sont devenues plus faibles et trop démodées. Les citoyens, en tant qu'individus, ne semblent pas avoir suffisamment de possibilités d'influencer ces institutions. »

Une commission gouvernementale sur l'économie a réitéré ce genre de critique des institutions de la protection sociale. Cette commission a vu le jour en $1992^{2}$; elle était chargée d'analyser les problèmes économiques émergeants en Suède et de proposer des directives pour la politique économique. La commission a accusé l'État providence d'être en partie responsable des problèmes actuels qui résultaient de l'expansion des dépenses publiques et des caractéristiques de l'organisation de la protection sociale. Selon la commission, les institutions de la protection sociale manquaient d'efficacité. Bref, la crise économique était aussi une crise de ces institutions. Ce qui était nécessaire, c'était une organisation sociale avec des "citoyens actifs", la "responsabilité individuelle», un " marché libre », et une " relation directe et très claire entre les efforts et les résultats ». Pour sortir de la crise, la notion de marché était considérée comme très importante et des réductions dans le secteur public étaient nécessaires.

On considérait que la liberté de choisir et la compétition entre des alternatives différentes avec la privatisation étaient une façon de moderniser des institutions qui fonctionnent mal dans l'État providence, y compris, dans l'éducation. Depuis longtemps la 
politique nationale d'éducation en Suède dépendait d'un certain consensus, de décisions prises par un pouvoir central et de solutions standardisées.

Après la Deuxième Guerre mondiale, on a introduit les réformes dans l'éducation dans un climat consensuel. Cependant, depuis le milieu des années soixante-dix, les critiques augmentent. On a critiqué l'école unique pour son manque d'efficacité et de direction. Les parents et les élèves se sont plaints, disant qu'ils avaient peu d'influence dans les écoles par rapport à d'autres institutions. La confiance publique dans l'éducation a diminué à partir de ces années-là. Ceci a été souligné dans le rapport de la commission d'État sur le pouvoir et la démocratie en Suède. Le manque d'efficacité du système éducatif a été davantage évoqué par la commission sur l'économie de 1993 :

- avec moins d'exigences détaillées concernant le contenu, les méthodes d'enseignement, l'organisation des écoles et avec une compétition entre écoles - aussi bien publiques que privées -, l'école unique suédoise serait certainement plus performante ;

22 - elle laisserait davantage de liberté de choix aux parents et aux élèves, cette liberté étant considérée comme un moyen d'améliorer les performances de l'éducation en Suède.

\section{La restructuration de l'enseignement}

Depuis le milieu des années soixante-dix, différentes façons de restructurer l'éducation ont été avancées sur le plan politique sous forme de décentralisation, etc. De telles mesures sont en augmentation depuis la fin des années quatre-vingt, associées à des demandes plus pressantes de réductions budgétaires. Cette période est marquée en Suède par des changements fréquents de majorité gouvernementale. Après plus de trente années d'hégémonie sociale-démocrate, la droite a pris le pouvoir en 1976 et a gouverné jusqu'en 1982. Ce sont les sociaux-démocrates qui ont gagné l'élection de 1982, mais en 1991 ils ont été remplacés par une coalition de droite menée par les conservateurs. En 1994, la gauche a gagné les élections et les sociaux-démocrates ont formé le gouvernement.

Murray $^{3}$ a étudié l'effet de ces changements sur la politique de l'éducation en Suède. Selon lui, au départ, les changements politiques n'étaient pas accompagnés de changements plus radicaux dans la politique de l'éducation. De plus, Murray considère que, dans le domaine de l'éducation, les sociaux-démocrates avaient préparé le chemin pour les conservateurs quand ils sont arrivés au pouvoir en 1991. Les sociaux-démocrates avaient décentralisé la structure de prise de décision, avaient déréglementé les transferts d'argent et ouvert la voie pour les écoles indépendantes et la privatisation de l'éducation.

\section{La course à la restructuration des conservateurs}

La rupture idéologique qui s'est opérée quand les conservateurs et leur coalition avec le parti libéral et le parti centriste sont arrivés au pouvoir en 1991 est particulièrement intéressante. Les conservateurs avaient élaboré une nouvelle stratégie. Ils n'étaient plus intéressés par le consensus, mais plutôt par le fait de mener une course. Le ministre de l'Éducation, tout comme le ministre des Affaires scolaires, venait du parti conservateur. Une course de vitesse a commencé par un certain nombre de décisions et de commissions « turbo ». De cette période, Murray ${ }^{4}$ a déclaré : 
26 «Il y a un contraste très net entre le gouvernement de droite de 1991-1994 et le gouvernement conservateur de 1976-1982. Il ne s'agissait pas simplement d'une continuation de la politique sociale-démocrate, mais d'un emploi habile de la situation, ainsi que d'une utilisation très efficace de leur mandat limité à trois ans ».

On pense que la qualité de l'éducation va être améliorée par la compétition dans un marché où des consommateurs bien informés feront des choix parmi des écoles déréglementées qui ont obtenu la possibilité de développer leur propre projet.

Dans un rapport récent, les rapporteurs de l'OCDE $^{5}$ considèrent la politique nationale de l'éducation des conservateurs comme un changement radical de direction. Ils remettent en question ce changement de plusieurs façons et en soulignent le manque de réflexion. La déréglementation et les choix créent de nouveaux problèmes; les intérêts nationaux dans le développement des ressources humaines sont presque négligés par cette politique.

29 La période de 1991-1994, avec un gouvernement conservateur, est dynamique quand on considère les changements dans la politique nationale de l'éducation en Suède, au moins sur le plan de la formulation.

\section{Retour des sociaux-démocrates au pouvoir}

Quand les sociaux-démocrates sont arrivés au pouvoir à l'automne 1994, ils avaient critiqué sévèrement la politique d'éducation des conservateurs pendant leur campagne électorale et, entre autres, ils avaient promis de supprimer les chèques-éducation. Cependant, le nouveau ministre social-démocrate des écoles a hésité et il n'a fait que baisser leur portée de $85 \%$ à $75 \%$ du coût moyen des études des élèves. Un certain nombre de commissions d'État ont été mises en place mais, pour le moment, il y a eu peu de résultats. Leurs objectifs se retrouvent dans une circulaire. Tout en rappelant le fait que l'école est au service de la communauté ainsi que le rôle des parents et des élèves dans les décisions des écoles, on insiste aussi sur l'équité, l'égalité et sur le fait que l'éducation doit favoriser l'intégration sociale. Dans une note du 24 avril 1995, on trouve des critiques du système actuel des chèques-éducation et on évoque une révision des règles qui s'appliquent aux écoles indépendantes. Dans cette même note, on écrit :

«On espère que les écoles municipales deviendront de plus en plus sensibles aux demandes et aux intérêts des élèves, des parents et du personnel scolaire qui font actuellement le choix d'une école indépendante au lieu de rester dans les écoles municipales. »

31 Un an plus tard le ministre a abandonné le taux fixe pour le chèque-éducation de $75 \%$ et maintenant, il y a une négociation entre chaque autorité locale et les écoles indépendantes existantes. Qu'en sera-t-il à l'avenir? La déréglementation et la privatisation des écoles vont-elles se poursuivre? Ou y aura-t-il une rupture avec la politique de l'éducation des conservateurs?

\section{Quel est l'impact de la restructuration sur l'école unique?}

Ce qui s'est passé de 1988 à 1995 a entraîné beaucoup de changements dans les textes de loi sur l'éducation. Englund ${ }^{6}$ les a comparés aux tendances internationales et a trouvé que 
la Suède semblait en accord, sur le plan de la formulation, avec les autres mouvements de droite dans le monde en ce qui concerne la notion de marché et la privatisation. En Suède, la restructuration va de pair avec un changement de point de vue. Au lieu de considérer l'éducation comme un bien public, elle est devenue un bien privé et l'école, au lieu de former un élément d'un projet de société, est devenue l'arène de stratégies familiales de reproduction sociale. Cela veut dire que la restructuration n'est pas simplement une question de gestion, mais qu'il y a un lien avec le changement d'orientation de l'école unique en Suède.

Quand on a affaire à la restructuration, on se préoccupe aussi des conditions financières. La décentralisation et la déréglementation de l'éducation furent en grande partie le résultat de changements dans les prémices économiques sur le financement de l'éducation. On pensait améliorer les performances des écoles par les mesures adoptées. Toute décision concernant des réductions financières devait s'opérer au niveau local, puisque c'est à ce niveau que l'on sait le mieux où on peut économiser de l'argent. La raison annoncée de la restructuration était l'amélioration de la gestion.

$\mathrm{Au}$ cours des années quatre-vingt-dix, on a pu remarquer une baisse considérable des moyens alloués à l'éducation dans les écoles uniques. L'impact économique est alors très visible en termes de statistiques et sur le terrain. Il semble difficile de concilier une baisse des moyens avec de meilleures performances, mais, en tout cas, on a au moins réussi à faire des économies.

Quels furent les effets réels de ces ambitions? La politique a-t-elle laissé la place aux forces du marché? C'est là que les points de vue divergents quelque peu. Miron considère ces changements plus ou moins comme une révolution. Voici ce qu'il en dit :

«La décision prise en juin 1992 d'introduire des chèques-éducation comme moyen de distribution de fonds à des écoles indépendantes place la Suède dans le peloton de tête en ce qui concerne la liberté de choix et les forces de marché dans l'éducation. »

Lundahl ${ }^{8}$ est plus réticente. Elle remarque que la source de la politique de l'éducation s'est un peu déplacée, ainsi que le pouvoir et les mécanismes de contrôle. Mais elle ajoute :

«Bien que la droite annonce que le rôle de l'État devrait être réduit en faveur de la liberté de choix individuel, je pense que ce n'est pas la tendance dominante dans l'éducation, même s'il y a eu certaines avancées en ce sens. Quand la politique centralisée a échoué, on a donné aux autorités locales des compétences accrues pour la distribution des moyens et pour définir des objectifs pour l'éducation en accord avec la législation nationale sur l'éducation, le programme national et les directives. De nouveaux mécanismes de pouvoir et de contrôle de l'État ont remplacé les anciens et nécessitent probablement de nouveaux outils. »

Les rapporteurs de l'OCDE ${ }^{9}$ étaient plutôt sceptiques quant à la mise en place de la politique des conservateurs, en partie à cause du manque d'analyse professionnelle et en partie à cause de l'accueil très variable fait à cette politique au niveau local.

Cependant, selon l'ancien secrétaire d'État conservateur, Odd Eiken, l'impact a été considérable ; environ 10 à $25 \%$ des familles ont choisi une école autre que celle de leur secteur. Les sociaux-démocrates pensent que le pourcentage réel est plus faible. En fait, environ $2 \%$ ont choisi des écoles indépendantes. C'est seulement dans un nombre très restreint de communes que plus de $2 \%$ ont demandé une autre école que celle du secteur scolaire. Il faut comprendre qu'en Suède, sauf dans des villes avec une population dense, il n'y a pas de possibilité de choix étant donné les grandes distances entre les écoles. De plus, quoique cela soit difficile à prouver, jusqu'à présent, il n'y a pas eu une grande 
disparité dans la qualité des écoles (en comparaison, par exemple, avec certains secteurs des États-Unis) et peu d'entre elles ont un profil spécifique. Il se peut que la tradition du programme national en Suède fournisse une explication partielle sur ce point. Nous pensons donc que plusieurs éléments vont à l'encontre de l'opinion du secrétaire d'État conservateur. Jusqu'à présent, la liberté de choix n'a pas déclenché de révolution dans les écoles en Suède.

Quant à la notion de marché dans l'éducation de Roger Dale, il faut penser que, pour un certain nombre de raisons, l'éducation ne correspond pas aux critères d'un marché ; c'està-dire que les mécanismes de retour d'information ne fonctionnent pas convenablement. Bref, on peut considérer la restructuration en Suède jusqu'à présent comme un échec en termes de marché. La mise en place d'un certain choix individuel et de la compétition entre les écoles n'a pas donné lieu à un système de retour d'information qui fonctionne bien.

$\mathrm{Au}$ cours de nos visites dans les écoles et de nos entretiens avec les enseignants et les directeurs, nous nous sommes rendus compte que la possibilité de choisir l'école est sujet à discussion pour les parents et les élèves. Dans une certaine mesure, les directeurs ont l'impression d'être des gestionnaires plutôt que des enseignants. Pour citer l'un d'entre eux :

"Je suis devenu un gestionnaire. J'ai la responsabilité de remplir les salles de classes et de faire en sorte que cela marche financièrement. Ainsi nous devons trouver des alternatives attrayantes comme, par exemple, le patinage pour les filles et le hockey sur glace pour les garçons. »

41 Les enseignants ont peur de perdre leurs élèves et que leur école se retrouve en perte de vitesse. Mais, en réalité, le nombre réel d'élèves qui choisissent une autre école que celle des environs est réduit. On pense que la compétitivité entre écoles varie beaucoup. À quelques exceptions près, il s'agit d'écoles qui se trouvent dans certains quartiers des villes. Mais, pour le moment, il n'y a pas de compétition qui ait des effets tels que cela entraîne des mouvements d'élèves.

Dans beaucoup de régions, l'introduction de la notion de marché n'a pas eu de répercussions. En dehors des plus grandes villes, très peu de familles ont utilisé, ou ont pu utiliser, la possibilité de choisir une école autre que celle du secteur. En réalité, nous pensons que les gouvernants n'ont jamais vraiment mis en pratique la loi du marché en Suède. Mais les politiques (une combinaison entre les décisions prises par les sociauxdémocrates et celles des conservateurs) ont déréglementé l'éducation, ont ouvert la voie aux réductions financières et ont déclenché une transformation de l'éducation pour faire en sorte qu'elle devienne un bien privé plutôt que public. Les préoccupations individuelles des familles ont remplacé, dans une certaine mesure, les décisions politiques. Et, par la suite, cela peut entraîner, subrepticement, d'autres changements dans la politique de l'éducation.

Cependant, l'augmentation des réductions budgétaires, associée à une différenciation plus grande entre les municipalités, pourrait donner lieu à des répercussions plus grandes de la restructuration de l'éducation en ce qui concerne la compétition entre les écoles. Surtout dans un contexte de changement d'orientation : d'un projet collectif à un projet individuel, du public vers le privé et ainsi de suite. Cela pourrait signifier le démantèlement de l'école unique en Suède. Cela implique aussi que la Suède se conforme à l'approche d'un certain nombre d'autres pays où la notion de marché a été introduite. 

années soixante aux années quatre-vingt, quoiqu'il y ait des tendances en ce sens dans le débat public. On va peut-être aller vers un modèle d'éducation qui serait un droit du citoyen et aussi un projet collectif, avec des possibilités accrues pour s'exprimer ${ }^{10}$ Selon Rothstein ${ }^{11}$, notre manière de gérer l'éducation est une question politique. L'analyse de celle-ci offre un moyen de comprendre le sens de la politique de l'éducation. Pour nous, la question de la participation des élèves dans les orientations, non pas en tant que consommateurs mais en tant que citoyens en formation, est une troisième voie.

Pour conclure, l'emploi de la loi du marché dans l'éducation, jusqu'à présent, semblerait plutôt un exemple de l'échec du marché. L'introduction de cette notion ne signifie pas la dépolitisation de l'éducation. Quand on voit les tâches de l'éducation dans la société moderne, il y a peu de raisons de penser que l'État va réduire son rôle. On peut s'attendre à l'inverse, mais on ne sait pas quelle sera la nature de ce rôle.

\section{NOTES}

1. Demokrati och Makt i Sverige. Maktutredningens huvudrapport, Stockolm, Allmaänna förlaget (commission d'État sur le pouvoir et la démocratie), SOU, 1990, p. 190.

2. Nya villkor för ekonomi och politik, Ekonomikommissionens förslag, Stockholm, Fritzes (commission gouvernementale sur l'économie), SOU, 1993, 16.

3. M. Murray, « Fran fullbordan till upplösning och... ? Svensk skolpolitik 1980-1995 », Didactica Minima, 35 (4), 1995, p. 6-27.

4. Op. cit.

5. OCDE, Reviews of National Policies of Education: Sweden, Paris, OCDE 1995.

6. T. Englund, «New International Trends for Swedish Schools - Marketization, Privatisation, Religiousization, Languagization...", in: Kallôs et Lindblad (eds), New Policy Contexts for Education: Sweden and United Kingdom, Report from the first comparative seminar, Report from Education Department at Umea University

7. G. Miron (éd.), Towards Free Choice and Market-oriented Schools. Problems and Promises, Skolverkets, rapport $n^{\circ}$ 2, Stockholm, IIE, 1993

8. L. Lundahl, Something Borrowed and Something New. The study of Education Policy: its Scope and Limite, Paper presented at the second comparative policy seminar: New Policy Contexte for Education: Sweden and England, Center for Educational Research, April 27-29 1995, Kings college, London.

9. Voir note 5 .

10. A. Hirschman, Exit, Voice and Loyalty, Cambridge, Harvard University Press, 1970.

11. B. Rothstein, Vad bôr staten gôra ? Om Välfärdsstatens moraliska och politiska logik, Stockholm, SNS, Forlag, 1994. 


\section{RÉSUMÉS}

Les fréquents changements de majorité en Suède, au cours des dernières décennies, amènent à s'interroger sur l'évolution de l'école unique et sur les effets d'une déréglementation très controversée, qui tend à ne plus faire de l'éducation un projet de société.

INDEX

Index géographique : Suède

Mots-clés : choix d'une école, réforme de l'enseignement, système éducatif

\section{AUTEURS}

\section{LEIF BRETTELL}

Chef de projet, département de formation interne, université d'Uppsala, Suède.

\section{SVERKER LINDBLAD}

Professeur, département d'éducation, université d'Uppsala, Suède. 